\title{
Use of fine needle aspiration cytology for investigating lymphadenopathy in HIV positive patients
}

\author{
E Martin-Bates, A Tanner, S K Suvarna, G Glazer, D V Coleman
}

\begin{abstract}
The cytological diagnoses of 27 lymph node aspirates were compared with the histological diagnoses or clinical outcome in 23 HIV positive patients. There was agreement between the cytological and histological diagnoses in 14 of the 16 surgically biopsied cases. The clinical outcome in the remaining 11 cases was consistent with the cytodiagnosis. Fine needle aspiration (FNA) is a reliable, minimally traumatic, cost effective method with high specificity. It is suitable for an initial rapid diagnosis in HIV positive patients with lymphadenopathy.
\end{abstract}

$(\Im$ Clin Pathol 1993;46:546-566)

HIV positive patients and those with AIDS often present with lymphadenopathy. ${ }^{1}$ The most common conditions affecting the lymph nodes in HIV positive patients are: reactive changes, including progressive generalised lymphadenopathy (PGL); opportunistic infections with Mycobacterium tuberculosis, Mycobacterium avium intracellulare, Histoplasma capsulatum and Cryptococcus neoformans; and malignant conditions such as non-Hodgkin's and Hodgkin's lymphomas and Kaposi's sarcoma. Clinical examination and radiological investigations on their own have diagnostic limitations. Histological analysis of a biopsy specimen can provide a reliable diagnosis but the method has several drawbacks. It is time consuming, elaborate health precautions must be observed in the operating theatre, and often the patients may be too ill for surgical intervention. Finally, the increasing number of HIV positive patients in recent years has posed an additional strain on theatre time, budget, and resources.

We studied the accuracy of fine needle aspiration (FNA) of lymph nodes as an alternative diagnostic method to histological examinations. This method is being used with increasing frequency in clinical practice because it is less expensive than surgical biopsy, less time consuming, and can be tolerated easily by the patients. Moreover, aspiration can be carried out in an outpatient setting or at the bedside. ${ }^{1-5}$

The cytological diagnoses of the FNA material were compared with the histological diagnoses from the surgically removed lymph nodes or with the clinical outcome in the cases where a biopsy specimen was not available.
Methods

Twenty seven fine needle aspirates were obtained from $23 \mathrm{HIV}$ positive patients who presented with lymphadenopathy.

The palpable lesions were aspirated in an outpatient setting, without anaesthetic. A $10 \mathrm{ml}$ syringe and 23 gauge needle were used and several passes were made. The hand used to immobilise the lesion was protected by a special Kevlar glove (Du Pont, Geneva, Switzerland), which has needle resistant padding on the finger tips. The gloved hand was moved away before the needle was withdrawn from the lesion. No mask was worn, but special care was taken in expelling the aspirate immediately and very closely to the surface of the labelled glass slides to prevent the creation of an aerosol. The material was spread evenly with another slide. All slides were air dried and fixed with methanol. The needle was rinsed with saline and the content was expressed into a test tube for making further slides if required. The slides, needle washings, and request form were sent to the laboratory in a double pocketed plastic bag bearing a health hazard sticker. The stains routinely used in the laboratory were MayGrünwald Giemsa and Grocott's. Immunocytochemical stains were performed when required. These included CAM 5.2 (Dako), LCA (CD45) Dako; L26 (Dako); UCHL1 (Dako); $\kappa$ and $\lambda$ (Dako). At the start of the study a Ziehl-Neelsen stain was done only when tuberculosis was suspected clinically or seen on light microscopic examination. Later specimens were routinely stained by the Ziehl-Neelsen method.

The histological material was routinely stained with haematoxylin and eosin Ziehl-Neelsen, and Grocott stains. Immunohistochemical stains were done when required.

All histological and cytological diagnoses were reviewed (by EMB and KS). The relevant clinical data were obtained from the request forms or, where available, from the clinical notes.

\section{Results}

Twenty seven lymph node aspirates were obtained from $23 \mathrm{HIV}$ positive patients-22 male homosexuals and one female heterosexual of African origin. The average age at first clinical presentation was 36.4 (range 19 to 72) years. Four patients presented with lymphadenopathy on more than one occasion.

Twenty six aspirates were from palpable 
Correlation between cytological and histological diagnoses and clinical outcome

\begin{tabular}{lcccl}
\hline Cytological diagnosis & & $\begin{array}{l}\text { Confirmed } \\
\text { on clinical } \\
\text { follow up only }\end{array}$ & $\begin{array}{l}\text { Histological } \\
\text { confirmation }\end{array}$ & $\begin{array}{l}\text { Other } \\
\text { histological } \\
\text { diagnoses }\end{array}$ \\
\hline Reactive changes & 11 & 4 & 5 & $2 \ddagger$ \\
Tuberculosis & 6 & 2 & 4 & 0 \\
Other infections & $4^{\star}$ & 4 & 0 & 0 \\
Malignant lymphoma & $2 \dagger$ & 0 & 2 & 0 \\
Kaposi's sarcoma & 4 & 1 & 3 & 0 \\
Total & 27 & 11 & 14 & 2 \\
\hline
\end{tabular}

${ }^{\star}$ Cryptococcus $n=1$; †Hodgkin's lymphoma $n=1$; $¥$ Tuberculosis; Histoplasma $n=1$; nonHodgkin's lymphoma $n=1$; Acute abscesses $n=1$. lesions; one aspirate was from an intraabdominal mass taken under computed tomographic guidance. The site of the lymph node aspirates were: cervical $n=14$; axillary $\mathrm{n}=4$; inguinal $\mathrm{n}=7$; submandibular $\mathrm{n}=1$; and intra-abdominal $\mathrm{n}=1$.

The cytological diagnoses of the 27 aspirated cases were: reactive changes $n=11$; tuberculosis $n=6$; other infections (one cryptococcosis, one histoplasmosis, two acute abscesses) $n=4$; malignant lymphomas (one non-Hodgkin's lymphoma, one Hodgkin's lymphoma) $n=2$; Kaposi's sarcoma $n=4$ (table).

Sixteen corresponding histological lymph node biopsy specimens were available for comparison. The histological diagnosis agreed with the cytological diagnosis in 14 cases and comprised: five reactive changes, four tuberculosis, two malignant lymphoma (one non-Hodgkin's lymphoma and one Hodgkin's lymphoma) and three Kaposi's sarcoma. Disagreement between the cytological and histological diagnoses occurred in two cases, both reported as reactive changes on cytology and as tuberculosis on histology reports.

Eleven FNA cases were subjected to clinical follow up alone. In four of them extranodal histological analyses were available and were identical with the cytodiagnosis (one cryptococcosis, one histoplasmosis, one tuberculosis and one Kaposi's sarcoma).

\section{Discussion}

The cytological diagnosis of reactive changes also included the progressive generalised lymphadenopathy (PGL) seen in AIDS. The diagnosis of reactive changes was based on finding a heterogenous population of cells in the aspirate. These cells included a spectrum of small and large lymphocytes some of which were in mitosis. Fragments of reactive follicular centre cells, including tingible body macrophages, syncytia of dendritic reticulin cells, and some plasmocytoid cells were also seen.

PGL is defined as unexpected enlargement of two or more extra-inguinal lymph nodes of at least three months' duration in individuals at risk for AIDS. The histological appearance varies, depending on the duration of the lesion, with florid reactive hyperplasia in the early stage to advanced lymphocytic depletion and "burnt-out" follicle centres in the late stage. This was reflected in the cytology in some cases, but the changes of PGL could not always be differentiated from reactive lymph nodes with different aetiology. The FNA material obtained from lymph nodes with tuberculous lymphadenitis showed caseation necrosis or epithelioid granulomata identical in appearance with those seen in HIV negative patients. The granulomata were composed of syncytial aggregates of oval or fusiform epithelioid histiocytes with "bluntended" and sometimes bent or curved nuclei. Langhans' type giant cells were very occasionally seen. Definitive diagnosis of tuberculosis was made only when acid fast bacilli were demonstrated on Ziehl-Neelsen stain. A Grocott stain was also carried out on every case where material was available to exclude other organisms. In the absence of acid fast bacilli or other organisms, the presence of caseation necrosis or granulomas in lymph node aspirates in HIV positive subjects was reported as consistent with tuberculosis unless proved otherwise.

The specificity of the FNA cytology in our study was $87 \cdot 5 \%$. This compares favourably with a study by Bottles et al from San Francisco, the only similar study of which we are aware. ${ }^{1}$ The authors studied 121 lymph node FNAs from 113 men with AIDS. The cytological diagnoses included 60 hyperplasias, 24 non-Hodgkin's lymphoma, 21 mycobacterial infections, 12 Kaposi's sarcomas, one Hodgkin's disease, one giant cell carcinoma, one nasopharyngeal carcinoma, and one squamous cell carcinoma. Histological or microbiological confirmation of the cytological diagnosis was available in 59 specimens. The specificity of the cytology in these cases was $93 \%$. Five cases were reported on FNA as hyperplasia and as malignant on histology (Hodgkin's disease three cases, non-Hodgkin's lymphoma one case, and Kaposi's sarcoma one case). The review of the cytological material showed no evidence of malignancy and therefore these five false negative results were interpreted as sampling error.

In our study two cases were reported on cytology as non-specific reactive changes alone (including PGL). Histological examination showed these to be tuberculosis (false negative rate $12.5 \%$ ). The aspirate of the first case, which was performed at the early stages of the study, contained insufficient material for carrying out special stains. The second aspirate probably represented a sampling error as no acid fast bacilli were shown on the Ziehl-Neelsen stain. The false negative rate reported by Bottles et al in the San Francisco study was $10 \%,{ }^{1}$ which is similar to ours.

No false positive results were reported in our study and no malignancy was missed. Our diagnostic difficulties were initially mainly in the area of differentiating tuberculosis from reactive changes which were later remedied by ensuring that sufficient material for routine staining for acid fast bacilli was available in every case. We therefore recommend routine staining for acid fast bacilli on 
every lymph node aspirate from HIV positive patients.

Bottles draws attention to the possibility of sampling errors in the cases of Hodgkin's disease where FNA may miss the affected part of the lymph node. ${ }^{1}$ Classic Reed-Sternberg cells and Reed-Sternberg cell variants are required for the diagnosis of Hodgkin's disease. In our only case of Hodgkin's disease occasional classic Reed-Sternberg cells were recognised and these allowed the correct diagnosis to be made. We agree, however, that if there is a discrepancy between the cytological diagnosis of non-specific reactive changes and clinically suspicious lesions for malignancy or infection, a repeat aspirate or surgical biopsy should be performed. Close communication between the pathologist and the clinician is essential.

In our series Kaposi's sarcoma did not present us with major diagnostic difficulties. All four cases of Kaposi's sarcoma had similar morphology of tightly packed rather monotonous atypical elongated cells with overlapping nuclei, and showing little pleomorphism and mitotic activity. ${ }^{5}$

Of the 11 cases that had had only clinical follow up at the time of the lymph node aspiration, four cases had identical extra-nodal pathology. In the remaining seven cases the cytological diagnosis was accepted as definitive. The clinical confidence in the cytological diagnosis increased with time and this led to a subsequent reduction in the number of biopsies performed. There are limitations to the FNA method, however, such as sampling error, which may result in false negative results. These diagnostic limitations could be overcome in many cases by close communica- tion between cytopathologist and clinician. Repeat aspiration is advised when there is a discrepancy between the cytological diagnosis and the clinical impression. FNA does not entirely exclude the need for histological methods and in difficult cases biopsy is still advised.

Although FNA is a relatively simple procedure we emphasise that certain precautions should be observed especially when dealing with HIV positive patients. The aspirator should wear a gown and special gloves to avoid needlestick injuries. Care must be taken in obtaining and transporting the specimens to the laboratory according to standard health regulations for dealing with hazardous material.

In conclusion, we recommend the fine needle aspiration (FNA) of lymph nodes in HIV positive patients as a reliable method for the diagnosis and follow up of cases with lymphadenopathy. The method is accurate, costeffective and acceptable to patients.

1 Bottles K, McPaul L W, Volberding P. Fine-needle aspiration biopsy of patients with the Acquired aspiration biopsy of patients with the Acquired Immunodeficiency Syndrome (AIDS): Experience

2 Shapiro A L, Pincus R L. Fine-needle aspiration of diffuse cervical lymphadenopathy in patients with Acquired immunodeficiency syndrome. Otolaryngol Head Neck Surg 1991;105:419-21.

3 Finfer M, Perchick A, Burnstein D E. Fine needle aspiration biopsy diagnosis of tuberculous lymphadenitis in patients with and without the Acquired Immune Deficiency Syndrome. Acta Cytol 1991;35:325-32.

4 Kragel P J, Miller L K, Casafrancisco D. Aspiration cytology of lymphocyte-depleted Hodgkin's lymphoma in a ogy of lymphocyte-depleted Hodgkin's lymphoma in a man infected with the human immunodefic

5 Hales M, Bottles K, Miller T, Donegan E, Britt-Marie L. Diagnosis of Kaposi's sarcoma by Fine-needle aspiration biopsy. Am $\mathcal{f}$ Clin Pathol 1987;88:20-5. 